\title{
Evaluation of the electrostatic association between cationic and stealth liposomes with nucleic acids in microfluidics
}

\section{Leonardo Jose Montebugnoli, Ismail Es, Lucimara G. de la Torre}

\begin{abstract}
Lipids can be used to create vectors for delivery of drugs, as well as genetic material. This work focused on the production of complexes between liposomes (with and without polyethylene glycol, PEG) and plasmidial DNA and small interfering RNA. Two mechanisms of production (complexation) were explored, one forms lipoplexes and lipid nanoparticles. Both structures formed were very different, resulting in distinct physical-chemical properties.
\end{abstract}

\section{Key words:}

Microfluidic, Gene Therapy, Complexation

\section{Introduction}

Phospholipids have the property of self-assembly when they are put in contact with a polar medium, due it's natural amphiphilic behavior, forming liposomes and other structures. When cationic lipids are used vesicle can interact more with cell-walls and genetic material, as both have an anionic characteristic ${ }^{1}$.

Lipoplexes consists in the complex of genetic material and liposomes as they are attached electronically on each other's surface. To make a lipoplex it is necessary a two-step process, first manufacturing the liposome and then, in a second step, complexing it with the genetic material. Physical chemical

Searching to improve the process another structure was proposed. A lipid nanoparticle is a solid particle made when lipids and genetic material are complexed in one step. So, the self-assembly and the complexation occurs simultaneously.

A microfluidic device based in diffusion was used to perform the production of structures and it was intrinsic related to the formation of those.

\section{Results and Discussion}

The production of the complexes with pDNA was used to optimize the system to find the best conditions to apply to produce siRNA complexes.

A precipitation investigation was performed varying the proportion between the flows of water, lipids (or liposomes) and genetic material and the final concentration of the complexes in the exit of the channel.

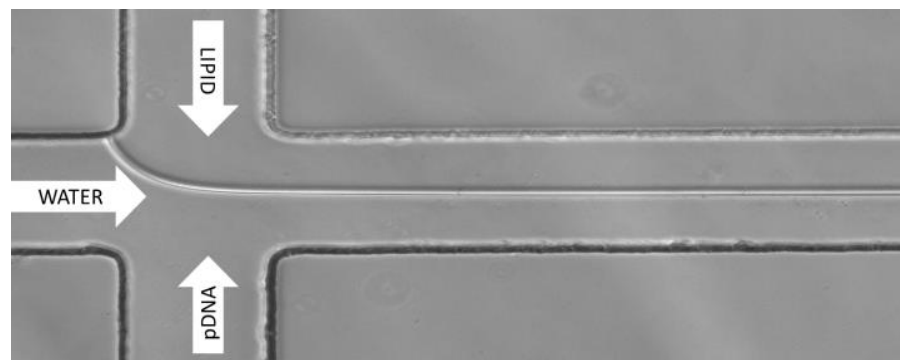

Figure 1 - Microfluidic device used to perform the complexations

This investigation led to the understanding of the system and it was observed that the best conditions were when the concentration of the lateral flows (which contains the genetic materials and the lipids or liposomes) were high and the central flow rate was also high. This offers a good resistance to the mass transfer resulting in good complexes. After this, the experiments with siRNA took place and the results can be seen in Table 1.

Table 1 - Physical-Chemical properties of the complexes

\begin{tabular}{|c|c|c|c|c|c|}
\hline \multirow{2}{*}{ Type } & Physical-Chemical Properties & LNP - pDNA & LPX - pDNA & LNP - siRNA & LPX - siRNA \\
\hline \multirow{3}{*}{ Conventional } & Z-average (nm) & $284 \pm 35$ & $187,4 \pm 0,4$ & $277,1 \pm 7,5$ & $202 \pm 1$ \\
& PDI & $0,22 \pm 0,01$ & $0,21 \pm 0,01$ & $0,14 \pm 0,02$ & $0,20 \pm 0,05$ \\
& Zeta Potential (mV) & $39 \pm 4$ & $58 \pm 1$ & $43 \pm 3$ & $40 \pm 2$ \\
\hline \multirow{3}{*}{ Stealth } & Z-average (nm) & $272 \pm 4$ & $172 \pm 4$ & $245 \pm 3$ & $199 \pm 2$ \\
& PDI & $0,58 \pm 0,03$ & $0,23 \pm 0,01$ & 0,184 & $0,19 \pm 0,03$ \\
& Zeta Potential (mV) & $55 \pm 1$ & $53 \pm 2$ & $46 \pm 2$ & $38 \pm 5$ \\
\hline
\end{tabular}

Although the structures of the complexes are quite different, it's observable that the PEG effect persists. The mean of the z-average of the particles with PEG is smaller than the conventional version. However, there is a discussion if the stealth effect will persist in the LNP, because the PEG will not be on the surface. This can be answered with transfection assay. The TEM was performed and can be seen in Figure 2.

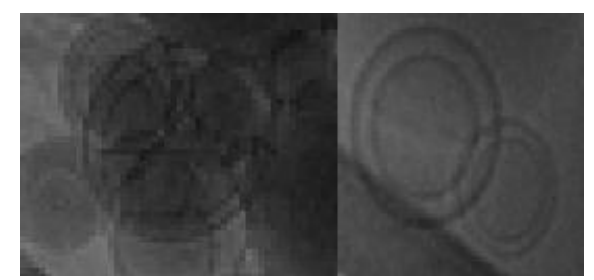

Figure 2 - TEM image for LNP (left) and LPX (right)

\section{Conclusions}

Both complexes were manufactured successfully, however it's necessary more experiments to determine which structure is better than the other for drug delivery purposes.

\section{Acknowledgement}

L. Montebugnoli acknowledges the financial support of the CNPq. Authors also acknowledges the CNPEM to the structure offered for perform part of this research.

${ }^{1}$ Balbino, T. A.; azzoni, A. R.; de la Torre, L. G. Microfluidic devices for continuous production of pDNA/cationic liposome complexes for gene delivery and vaccine therapy. Colloids and Surfaces B: Biointerfaces, v. 111, n. 0, p. $203-$ 210, 2013.

${ }^{2}$ Hood, R. R. et al.; "Microfluidic Synthesis of PEG- and Folate-Conjugated Liposomes for One-Step Formation of Targeted Stealth Nanocarriers". Pharm Res, V.30, n.1, p 1597-1607, 2013. 